Supporting Information for

\title{
Interfacial Engineering for Thermoresponsive Microgel Capsules: Polymeric Wetting versus Colloidal Adhesion
}

\author{
Xianyu Song ${ }^{1}$, Chongzhi Qiao ${ }^{1}$, Jiabo Tao ${ }^{1}$, Bo Bao ${ }^{1}$, Xia Han $^{2}$ and Shuangliang Zhao ${ }^{1, *}$ \\ ${ }^{1}$ State Key Laboratory of Chemical Engineering and School of Chemical Engineering, East \\ China University of Science and Technology, Shanghai, 200237, China \\ ${ }^{2}$ School of Chemistry and Molecular Engineering, East China University of Science and \\ Technology, Shanghai, 200237, China
}

*To whom correspondence should be addressed. Email: szhao@ecust.edu.cn 


\section{Artificial Neural Network Method}

The artificial neural network Method (ANN) aims to build a nonlinear heuristic model through multilayer perceptron (MLP). ${ }^{1}$ The MLP is composed of series of input layers, calculation centers or neurons (a single hidden in this study) and output layer. The nodes in the input layer describe all of required structure and external information (herein, system temperature, crosslink density and interaction strength). Similarly, the nodes in the output layer represent the predicted properties of interest (herein, contact angle). ${ }^{2}$ The connection between any two nodes has a unique weight that is randomly initialized and then is optimized during training process. The optimal numbers of hidden layers and nodes in a hidden layer have no universal values but are selected to maximize the efficiency and accuracy of ANN. ${ }^{3}$ Each node in the hidden layers consists of a computation function. The weighted input values, composed of all output values from the previous layer, will be transformed into the next layer. To achieve a best fit to pre-existing data, back-propagation algorithm, defined as the square of difference between the target and actual outputs, is utilized to compute the gradient of an error function. ${ }^{4}$ Then, the gradient descent updates the weights values for minimizing the error function. 
Table S1. Detailed structure information of microgel capsules.

\begin{tabular}{|c|c|c|}
\hline Crosslink density & Monomers $\mathrm{N}_{\mathrm{p}}$ & Cross-links $\mathrm{N}_{\mathrm{c}}$ \\
\hline 0.0158 & 20 & 404 \\
\hline 0.0217 & 15 & 948 \\
\hline 0.0310 & 10 & 2430 \\
\hline
\end{tabular}

Table S2. Training parameters in artificial neural network.

\begin{tabular}{|c|c|}
\hline Back-propagation algorithm & Levenberg-Marquardt \\
\hline Number of input layer & 3 \\
\hline Number of hidden layer & 1 or 2 \\
\hline Number of output layer & $1.0^{\mathrm{a}}$ or $0.1^{\mathrm{b}}$ \\
\hline Transfer function in hidden layer & 100 \\
\hline Leaning rate & 0.00004 \\
\hline Number of training & Hyperbolic tangent sigmoid \\
\hline Minimum error of training & \\
\hline
\end{tabular}

a The learning rate of 1 is adopted for achieving the linear correlations between simulation examples and different machine learning models. ${ }^{\mathrm{b}}$ The learning rate of 0.1 is adopted for predicting the properties of interfacial engineering.

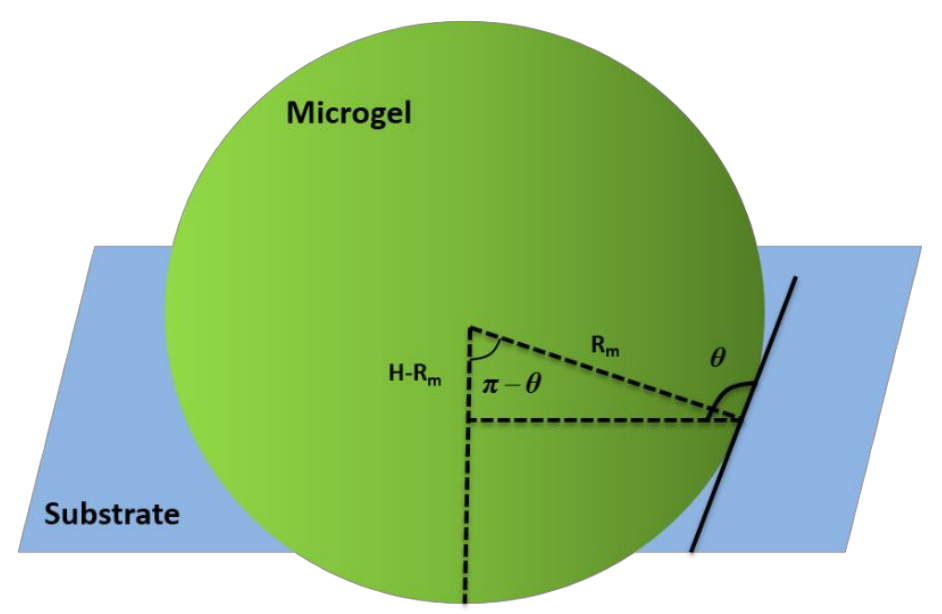

Scheme S1. Schematic illustration of the contact angle. 

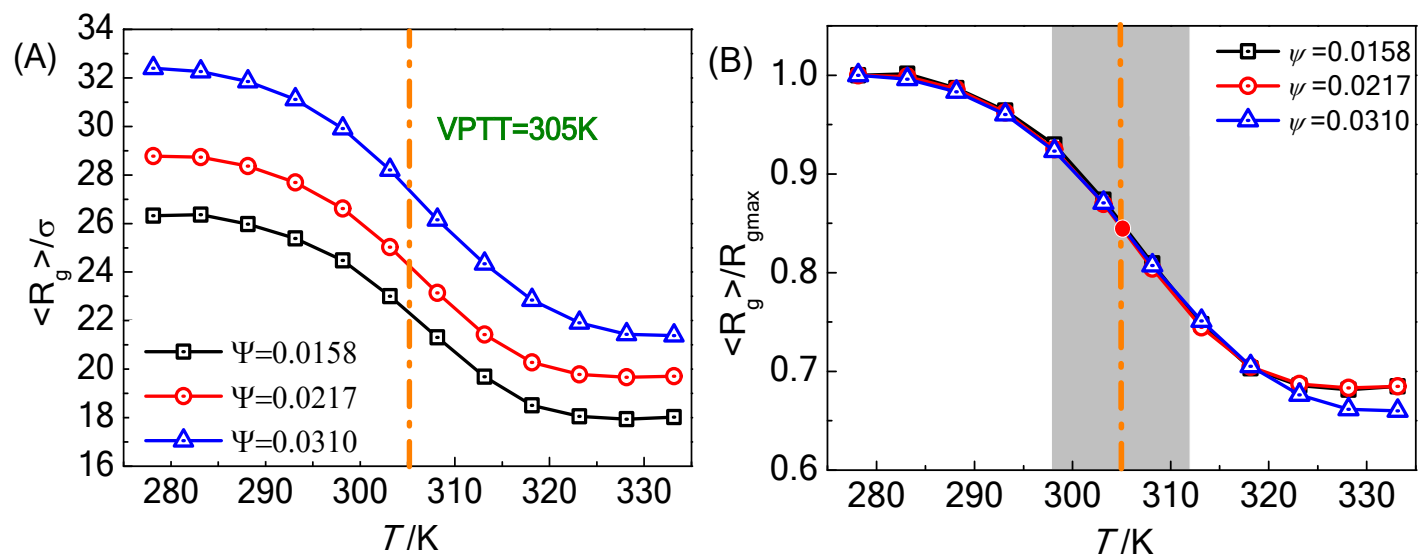

Figure S1. Verification of simulation results: (A) temperature-dependent radius-of-gyration and (B) $\left\langle R_{g}\right\rangle / R_{g \max }$ at the temperature ranging from 278 to $333 \mathrm{~K}$. The yellow lines correspond to the volume phase transition temperature (VPTT), while the gray zoom represents the VPTT zoom containing largest slope.
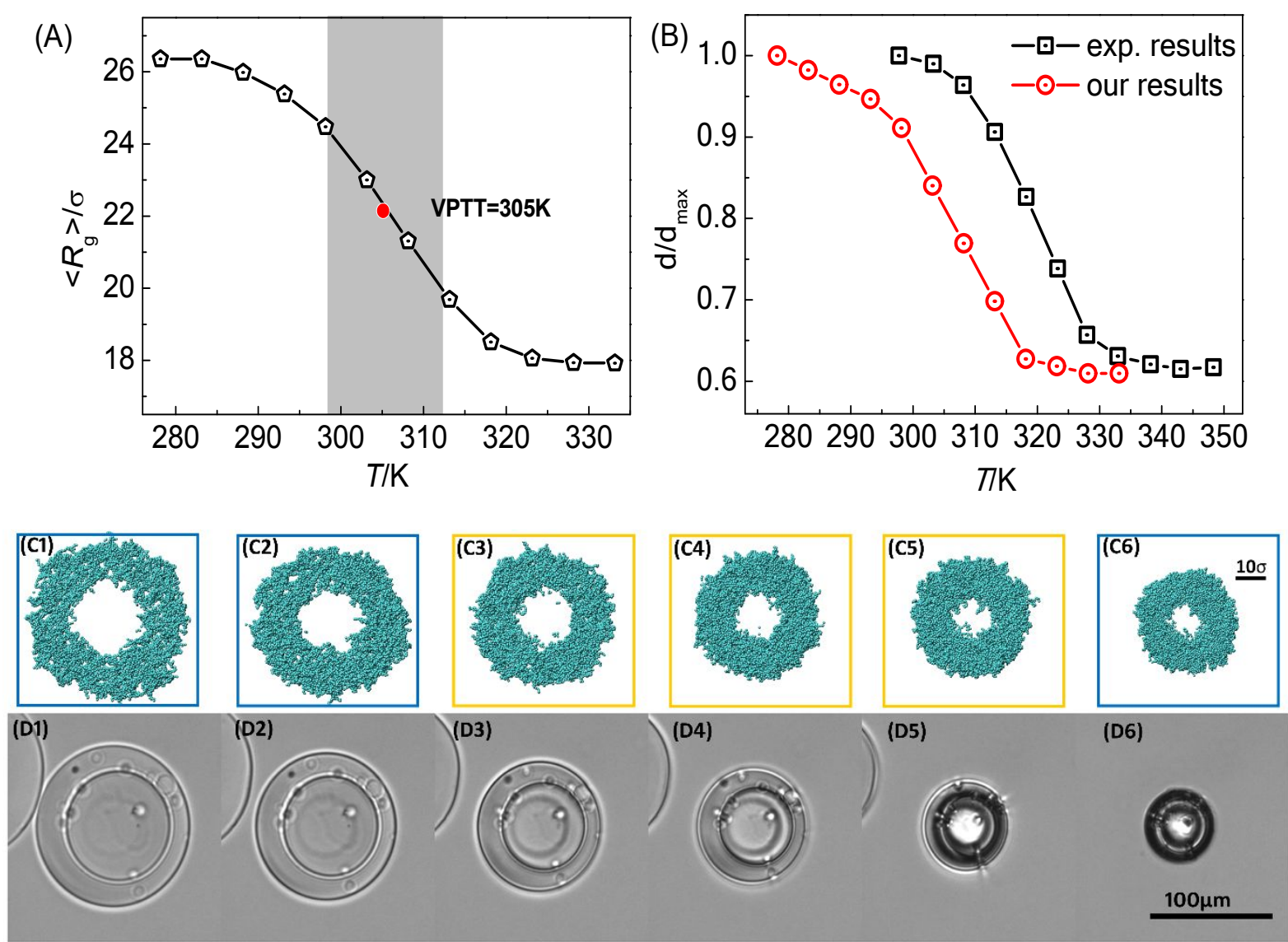

Figure S2. Variation of simulation results: ${ }^{5}$ (A) temperature-dependence of the simulated radius of gyration, (b) diameter of microgel capsule in terms of reduced system temperature 
predicted from our simulation in comparison with experimental results: (C1-C6) the representative simulation snapshots at different temperatures ranging from $298 \mathrm{~K}$ to $323 \mathrm{~K}$ with the crosslink density $\psi=0.0217$, (D1-D6) bright field microscope views of microgels heated from $300 \mathrm{~K}$ to $320 \mathrm{~K}$ by Wahrmund et al. ${ }^{6}$ The crosslink density of microgels for both figure (A) and (B) is $\psi=0.0158$.
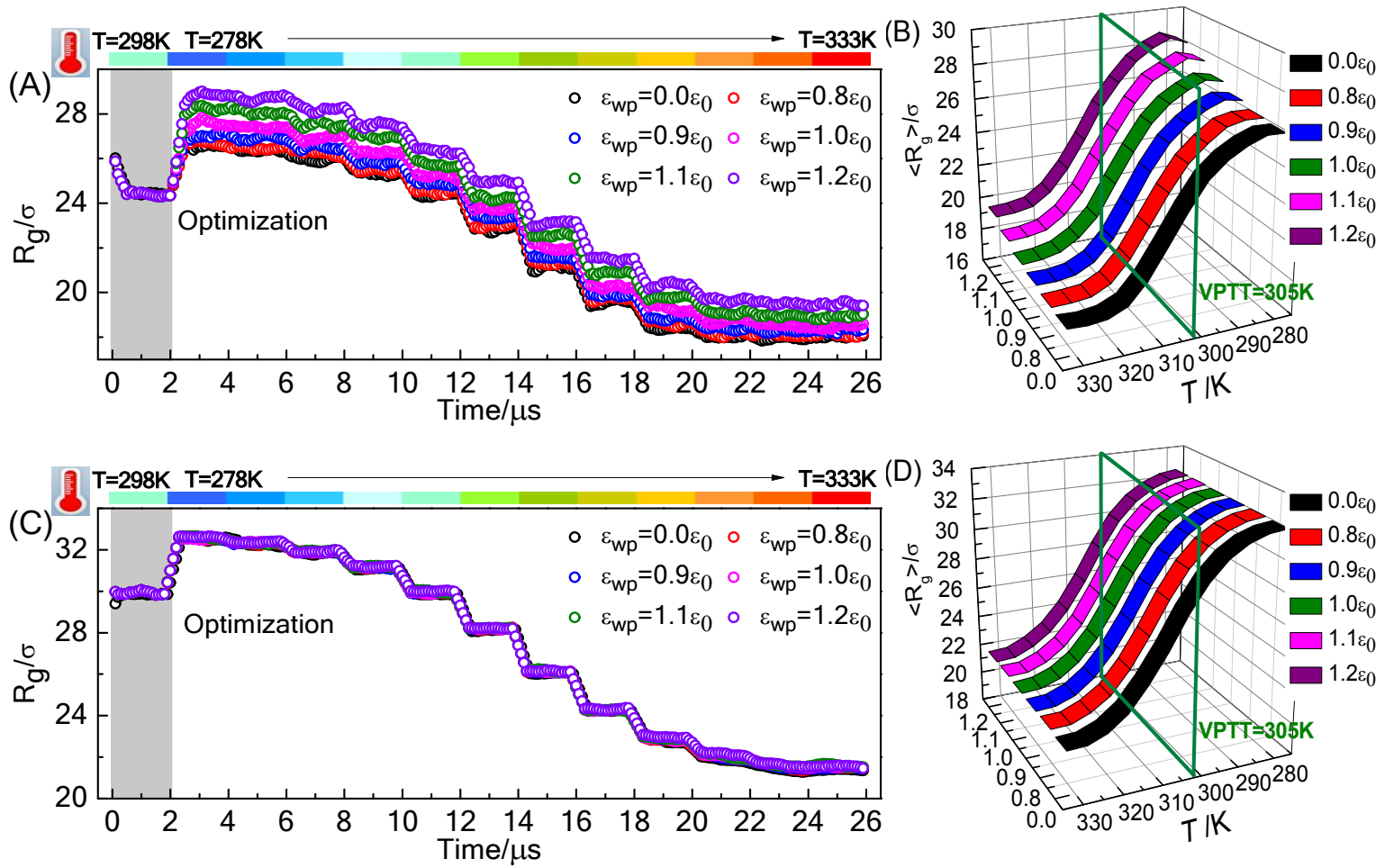

Figure S3. Conformational properties of polymeric chains for microgel capsules on the substrates: (A) and (C) radius-of-gyration for microgel capsules as a function of dynamics time at different interactions between the substrate and polymer, (B) and (D) mean radius-of-gyration for microgel capsules at different temperatures. The crosslink density is $\psi=0.0158, \psi=0.0310$, respectively. 

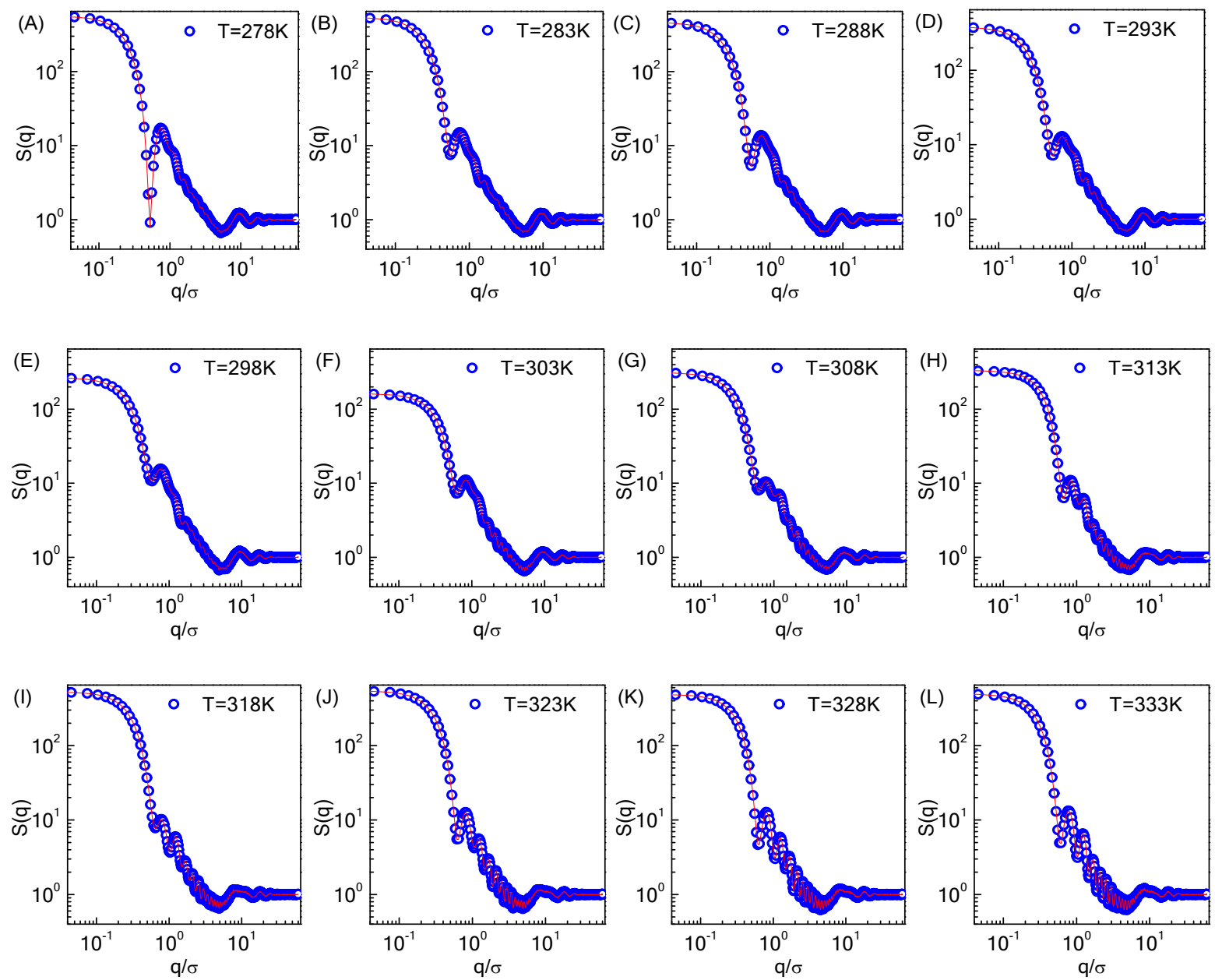

Figure S4. Static structure factor profiles of microgel capsules on the substrate with the temperature ranging from 278 to $333 \mathrm{~K}$. The crosslink density of microgels $\psi$ is 0.0158 . Interaction strength $\varepsilon_{\mathrm{wp}}=1.0 \varepsilon_{0}$.
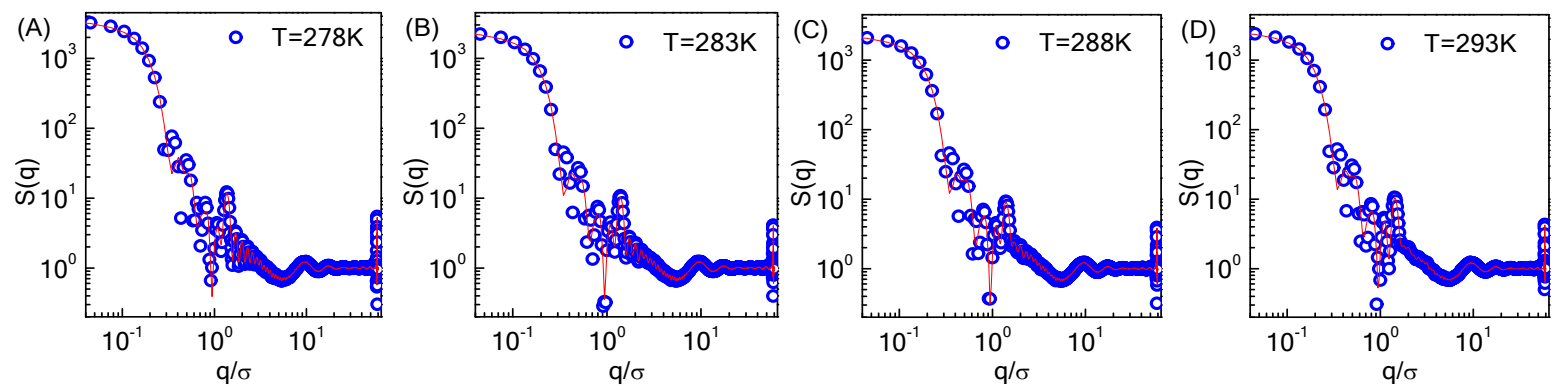

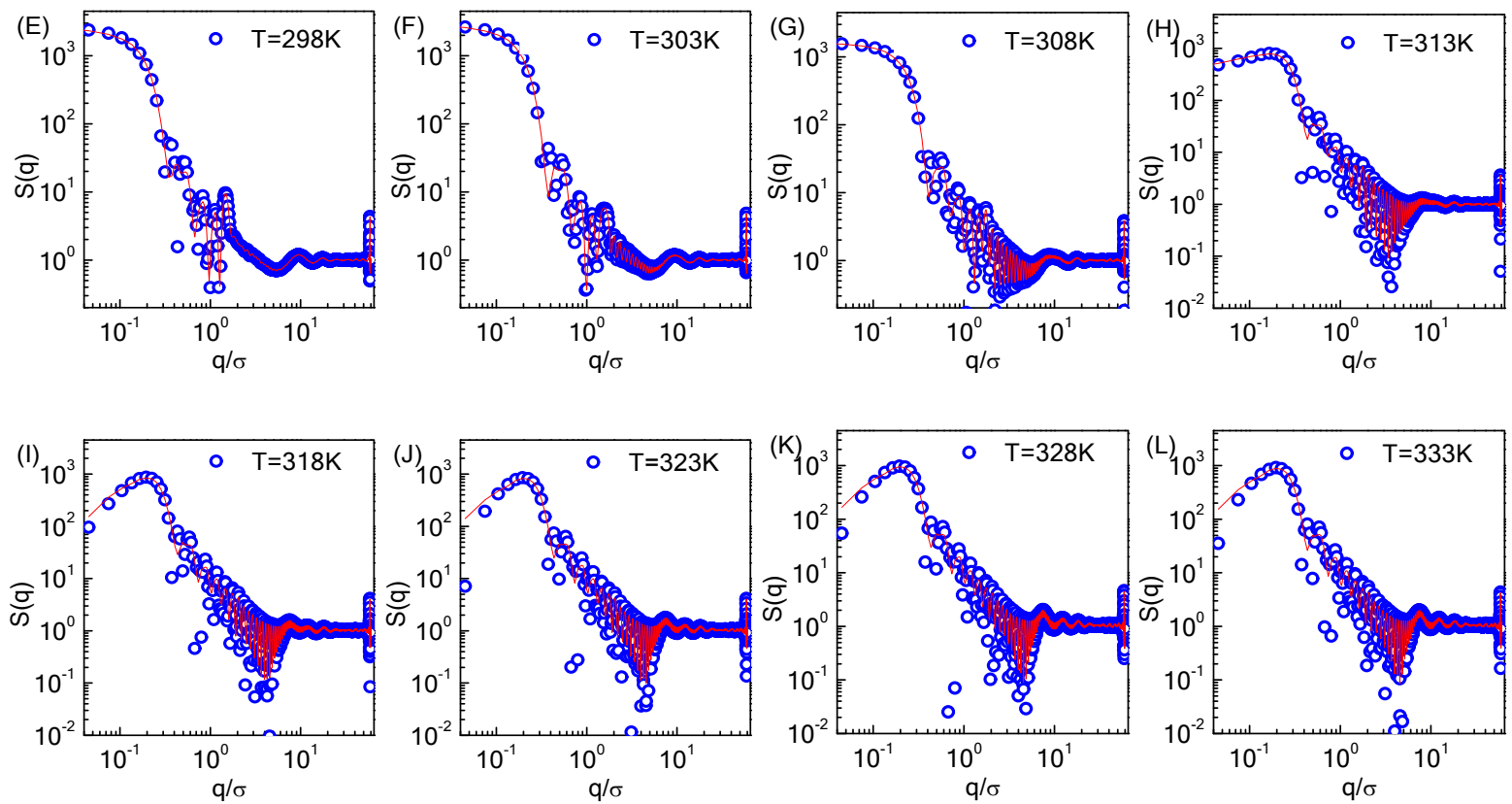

Figure S5. Static structure factor profiles of microgel capsules on the substrate with the temperature ranging from 278 to $333 \mathrm{~K}$. The crosslink density of microgels $\psi$ is 0.0310 . Interaction strength $\varepsilon_{\mathrm{wp}}=1.0 \varepsilon_{0}$.

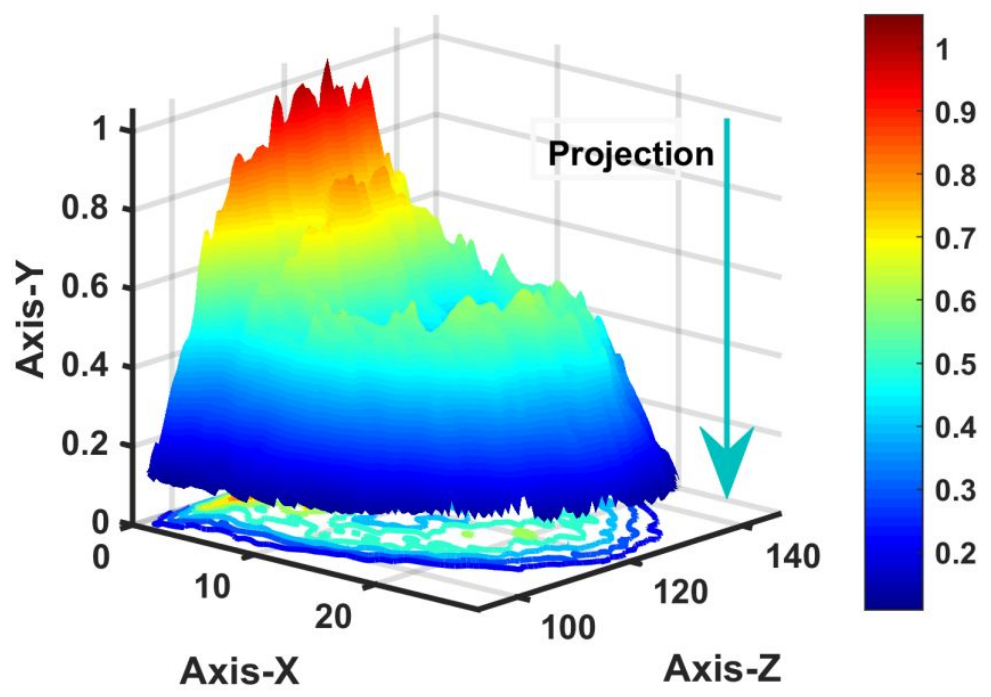

Figure S6. Schematic diagram for calculating the 2D contour of numeral density for the microgel capsules on the substrates. 


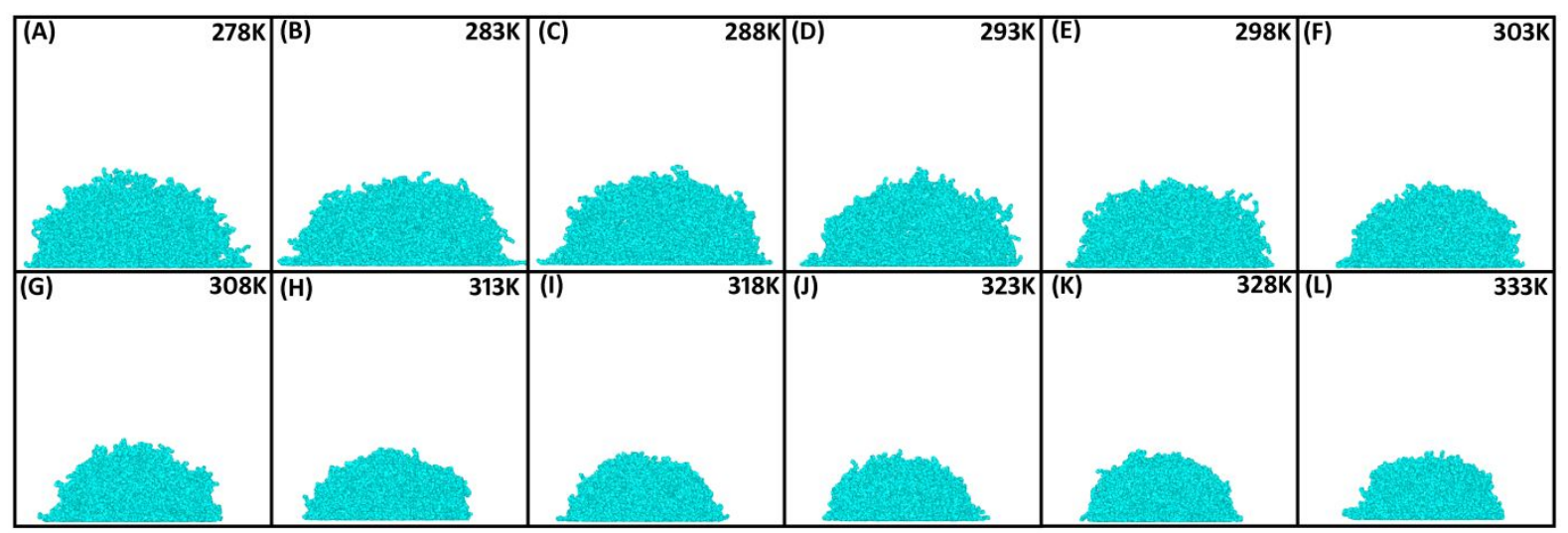

Figure S7. The representative snapshots of the microgel capsules on a solid substrate. The interaction between wall and polymer $\varepsilon_{\mathrm{wp}}=1.2 \varepsilon_{0}$, and the crosslink density of the microgel capsules is $\psi=0.0158$.

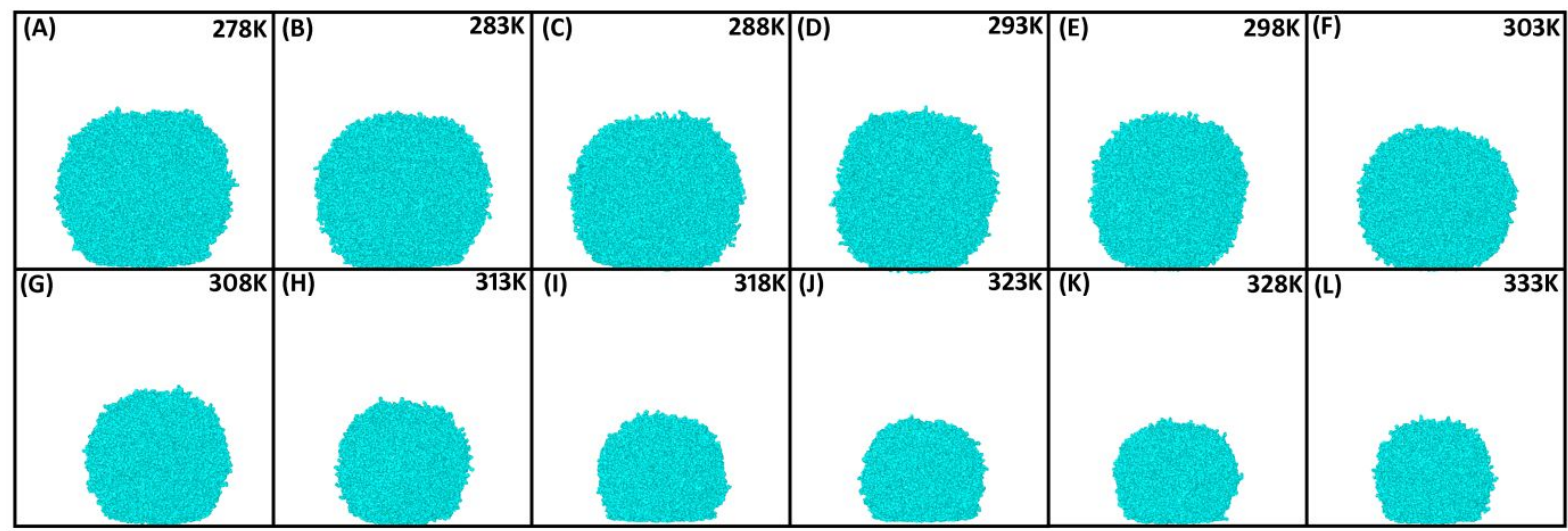

Figure S8. The representative snapshots of the microgel capsules on a solid substrate. The interaction between wall and polymer is $\varepsilon_{\mathrm{wp}}=1.2 \varepsilon_{0}$, and the crosslink density of the microgel capsules is $\psi=0.0310$.
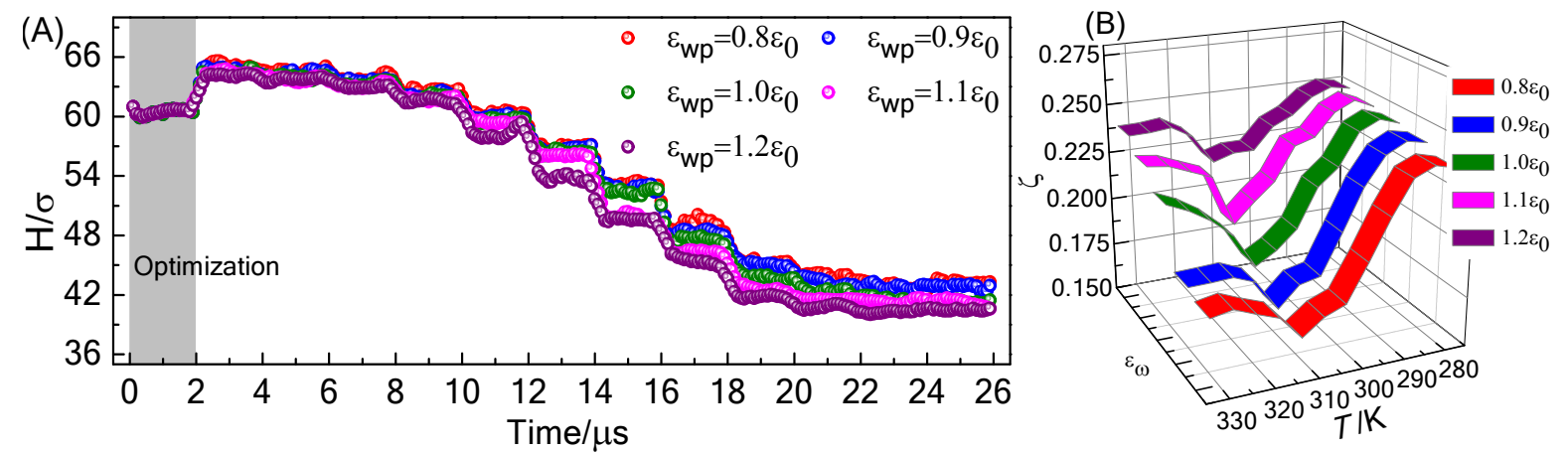

Figure S9. Structural characterization of microgel capsules on the substrates: (A) adsorption 
thickness, and (B) structural deformation parameter for the microgel capsules with crosslink density $\psi=0.0310$. The system temperature ranges from 278 to $333 \mathrm{~K}$.

\section{References}

1.Patra, T. K.; Meenakshisundaram, V.; Hung, J.-H.; Simmons, D. S., Neural-network-biased genetic algorithms for materials design: Evolutionary algorithms that learn. ACS combinatorial science 2017, 19 (2), 96-107.

2.Torrecilla, J. S.; Cancilla, J. C.; Aroca-Santos, R.; Pariente, E. S., Neural networks to estimate physicochemical properties of choline carboxylate ionic liquids. ACS Sustainable Chemistry \& Engineering 2015, 4 (2), 536-540.

3.Artrith, N.; Urban, A., An implementation of artificial neural-network potentials for atomistic materials simulations: Performance for TiO2. Computational Materials Science 2016, 114, 135-150.

4.Bhatt, A. K.; Pant, D., Automatic apple grading model development based on back propagation neural network and machine vision, and its performance evaluation. Ai \& Society 2015, 30 (1), 45-56.

5.Song, X.; Bao, B.; Tao, J.; Zhao, S.; Han, X.; Liu, H., Deswelling Dynamics of Thermoresponsive Microgel Capsules and Their Ultrasensitive Sensing Applications: A Mesoscopic Simulation Study. The Journal of Physical Chemistry C 2019, 123 (3), 1828-1838.

6.Wahrmund, J.; Kim, J.-W.; Chu, L.-Y.; Wang, C.; Li, Y.; Fernandez-Nieves, A.; Weitz, D. A.; Krokhin, A.; Hu, Z., Swelling kinetics of a microgel shell. Macromolecules 2009, 42 (23), 9357-9365. 